\title{
Introduction to Service Design for UX Designers
}

\author{
Virpi Roto \\ School of Arts, Design and Architecture, Aalto University, Espoo, Finland, virpi.roto@aalto.fi \\ Val Mitchell, Stuart Cockbill \\ School of Design and Creative Arts, Loughborough University, Loughborough, UK, \\ v.a.mitchell@lboro.ac.uk, s.cockbill@lboro.ac.uk \\ Jodi Forlizzi \\ Human-Computer Interaction Institute, Carnegie Mellon University, Pittsburgh, PA, forlizzi@cs.cmu.edu \\ Jung-Joo Lee \\ School of Design and Environment, National University of Singapore, Singapore, jjlee@ nus.edu.sg \\ Effie L-C Law \\ School of Informatics, University of Leicester, UK, lc19@1eicester.ac.uk
}

\begin{abstract}
We are witnessing the work of user experience (UX) designers expanding beyond single digital products towards designing customer journeys through several service touchpoints and channels. Greater understanding of the service design approach and the interplay between service design and UX design is needed by UX researchers and practitioners in order to address this challenge. This course provides a theoretical introduction to service design and practical activities that help attendees understand the principles of service design and apply key methods within their work. It is targeted at UX design practitioners, researchers, and students, and those with an interest in systemic approaches to design.
\end{abstract}

\section{CCS CONCEPTS}

- Human-centered computing Interaction design Interaction design process and methods

\section{KEYWORDS}

Service Design, User Experience Design, User Experience.

\section{Introduction}

In the past three decades, we have been witnessing a worldwide shift from a focus on product development to a focus on service delivery. The most profitable companies are now creating service offerings, known as "systems of interlinking services" [3]. Many companies are experiencing servitization, where digital services are added to existing products; others are experiencing productization, where physical products are added to service offerings [2]. The resulting product service ecologies are more complex, systemic, and data-driven than anything we have designed before [1],[4], leading to UX designers and researchers facing a range of inter-related challenges including:

1. Understanding and conceptualizing non-digital services in a digital form, such as the augmentation of taxicab services with on demand ride share platforms.

2. Delivering end to end experiences across parallel physical and digital touchpoints including, for example for the retail sector: advertising, branding, browsing of goods, payments, deliveries and returns, and backstage digitalization.

3. Co-designing with diverse stakeholders as business and design converge, and evaluation of success broadens beyond usability.

4. Navigating and defining roles where terminology is fluid and ambiguous at the interaction of Service Design and UX. 
Service Design (SD) is a holistic approach used to orchestrate the whole service journey considering customers, service providers and other relevant stakeholders. For user experience (UX) designers, who have often focused on the user interface design of a digital application, this service design perspective introduces new questions:

1. What does service design mean in practice?

2. What is the role and scope of UX design in a multi-stakeholder service design project?

3. Who is responsible for the user experience of the whole service journey?

4. How can UX and service designers best work together?

There is little research at the intersection of SD and UX design, and the research communities of these two areas are surprisingly separate. This course aims to encourage researchers to tackle the above questions that are now highly topical in industry. NORDICHI this year calls for greater awareness among HCI and UX practitioners of the impact on society of the experiences we shape and craft. This course will provide an understanding of how the tools and methods of SD can be used to provide a wider perspective to the stakeholder network around services, beyond the end user.

\section{Learning Outcomes}

As this course addresses both practitioners and researchers, we have designed the content to fulfil the needs of both. After this course, the practitioner attendees will be able to:

1. Work together with service designers through a better understanding of SD history, scope, process, and basic terminology.

2. Organize UX work to fit within a SD project by understanding the relation of UXD and SD and the possible roles of UX designers in SD projects.

The UX researcher attendees will be able to:

3. Conceptualize the relation between UXD and SD through a better understanding of SD history, scope, process, and basic terminology.

4. Identify topical research questions to tackle key challenges in the ongoing transformation of UX design towards more holistic service design and systemic thinking.

\section{Intended Audience}

The course is intended primarily for UX design practitioners, researchers, and students, and secondarily for attendees with an interest in systemic approaches to design.

As this is an introductory course, the participants are not expected to have any prior knowledge of service design. Basic understanding of UX design and user centric design processes will be helpful to understand the similarities and differences between SD and UX design.

\section{Content}

\section{Opening and introductions (10min)}

The course starts with introductions to the instructors and the course objectives. Depending on the number of the participants, participants may introduce themselves as well.

\section{History and the emergence of SD $(20 \mathrm{~min})$}

A short history of Service Design is presented to communicate the big picture. This covers concepts such as servitization and product-service systems. The contextual factors that have led to the emergence of SD are presented including the accelerating need for digitalization of existing service touchpoints, backstage digitalization, and the multiple channel options for each touchpoint. An introduction to SD theory will be provided including understanding of the nature of services, co-creation of services and how they are represented and constructed within a human centered design process.

\section{Practical Session, the basics of SD part I (60min)}

The first practical session will introduce core processes, methods and tools including stakeholder maps, customer journey mapping and service blueprinting, each supported by case study examples and exercises in small groups. SD terminology will be introduced on the way. 


\section{- Break -}

4. Practical Session, the basics of SD part II (30min)

Session 2 continues with the service design process, as described in the previous item.

\section{Interplay of SD and UXD (40min)}

Different scenarios of the interplay of SD and UX design throughout a design project will be presented, including typical project outcomes. The groups will discuss the appeal and relevance of the scenarios to their own situation.

\section{Conclusions \& Reflection (10min)}

We summarize the key messages and discuss the key take-aways with the participants.

\section{Service Design Clinic (10min)}

The instructors make themselves available for a SD clinic in the course room, to allow the participants to ask remaining questions, or ask for guidance related to their own practice or research.

\section{Practical Work}

The course consists of presentations by each of the instructors and exercises in small groups. Prepared templates and case study materials will be used to structure a series of activities to populate a stakeholder map and design a service blueprint for a customer journey pre-defined by the organizers. Examples of the groupwork outcomes will be presented back to the group with opportunity for reflection and discussion. In addition to asking the instructors directly, the participants can post questions and vote for others' questions to be responded via an online tool, e.g., Sli.do. This tool will be available also after the course and the instructors will use it to respond to as many questions as possible. Materials and content will be designed to be resilient to a shift to remote delivery if needed, for example through use of bespoke online mapping tools such as those provided by UXPRESSIA https://uxpressia.com

\section{Instructor Background}

Virpi Roto is Professor of Practice in Experience Design in Aalto University, Finland. She studies experience design in the intersection of UX and service design. Her methodological research aims to provide techniques for practitioners to improve user experiences. She was the main organizer of NordiCHI'16, CHI'18, and Nordes'19 workshops on the relation of UX and service design, and she has run UX courses and SIG sessions at CHI.

Val Mitchell is Programme Director of the UX Design MA at Loughborough University. Her research focusses on the development of transdisciplinary tools and methods for UX and SD and she has been a coorganizer of workshops at CHI2013 and UBICOMP14.

Stuart Cockbill specializes in teaching service design as part of the UX Design MA at Loughborough University. His research uses co-design processes to incorporate personal data within the design of future technologies and services.

Jodi Forlizzi is the Geschke Director and a Professor in the HCI Institute, Carnegie Mellon University, Pittsburgh, PA. Her current research interests include designing services in the form of educational games, assistive robots, social agents, and healthcare services. She is interested in the intersection of algorithms and human service delivery.

Jung-Joo Lee is a Deputy Head of Research and Assistant Professor in Division of Industrial Design, National University of Singapore, and a Director of Service Design Lab Singapore. Her research investigates roles of service design in organizational transformation and public-sector innovation. She was a co-organizer of workshops at CHI'15, CHI'18, and Nordes'19.

Effie Law is a full Professor in HCI, specialising in usability and UX methodologies. She was a coorganizer of NordiCHI'16, CHI'18 and Nordes'19 workshops on the relation between UX and Service Design. She was an instructor for the course on UX evaluation methods at CHI. 


\subsection{Sample Publications by the Instructors}

Virpi Roto, Jung-Joo Lee, Tuuli Mattelmäki and John Zimmerman. 2018, April. Experience Design meets Service Design: Method Clash or Marriage? In Extended Abstracts of CHI'18 (p. W26).

Alison Prendiville, Ian Gwilt, and Val Mitchell. 2017. Making sense of data through service design opportunities and reflections. Designing for Service: Key Issues and New Directions. 225-236.

Stuart A. Cockbill, Andrew May, and Val Mitchell. 2019. The assessment of meaningful outcomes from co-design: a case study from the energy sector. She Ji: The Journal of Design, Economics, and Innovation. $5,3: 188-208$.

Jodi Forlizzi \& John Zimmerman. 2013. Promoting Service Design as a Core Practice in Interaction Design. Proceedings of IASDR'13.

Jung-Joo Lee. 2020. Service design and blind mice, ACM Interactions, 27 (2), 20-21

\section{Resources}

Web site for the course to share relevant information and materials: blogs.aalto.fi/chisdcourse.

Service Design chapter by John Zimmerman and Jodi Forlizzi in the Encyclopedia of Human-Computer Interaction, 2nd Ed.

Our previous workshops on UX and SD: CHI'18 blogs.aalto.fi/xdsd, Nordes'19 blogs.aalto.fi/sdxd

\section{Promotional Strategy}

We will advertise the workshop in relevant mailing lists, such as CHI-Announcements, NordiCHIAnnouncements, and national HCI email lists, such as SIGCHI-Finland. In social media, the channels include NordiCHI, EstCHI, UXHel, and our wide network in social media. While NordiCHI is an academic conference, specific invitations will be sent to relevant personal contacts to attract industry participants.

\section{ACKNOWLEDGMENTS}

We thank Professors John Zimmerman and Tuuli Mattelmäki for research collaboration that made the preparation of this course possible. This work was partly supported by Business Finland, grants 7546/31/2016 and $81 / 31 / 2020$.

\section{REFERENCES}

[1] Jodi Forlizzi. 2018. The product ecology: Understanding social product use and supporting design culture. International Journal of design 2, 1.

[2] Luna Leoni. 2015. Servitization and Productization: two faces of the same coin? In Conference RESER.

[3] Ezio Manzini. 2014. Making things happen: Social innovation and design. Design Issues, 30, 1:57-66.

[4] Sangiorgi, D., \& Prendiville, A. (Eds.). 2017. Designing for Service: key issues and new

directions. Bloomsbury Publishing. 